Revista Chilena de Antropología 30/2 ${ }^{\circ}$ Semestre 2014 II6-120

\title{
Los Conjuntos Óseos de Cueva Milodón Norte 1, Lago Pueyrredón (Santa Cruz, Argentina)
}

\author{
Bone Assemblages from Cueva Milodón Norte 1, Lago Pueyrredón (Santa Cruz, \\ Argentina)
}

Mariana E. De Nigris ${ }^{\mathrm{i}}$

\begin{abstract}
RESUMEN
El objetivo de este trabajo es presentar los resultados de los análisis zooarqueológicos efectuados sobre los conjuntos óseos procedentes de las capas superiores de la secuencia estratigráfica del sitio Cueva Milodón Norte I (CMNI), lago Pueyrredón, noroeste de Santa Cruz, Argentina, fechadas en ca. 1.900 años AP. Los análisis realizados señalan similitudes y diferencias con otros conjuntos óseos procedentes de otros sitios de la región con cronologías similares. Se observa, en general, una baja diversidad de especies representadas, con un dominio absoluto del guanaco (Lama guanicoe). En cuanto a la representación de sus partes esqueletarias se registra una mayor frecuencia de elementos apendiculares y una baja proporción de costillas, patrón que no parece ser explicado completamente por la acción de procesos dependientes de la densidad ósea. La elevada diversidad de marcas de procesamiento y sus altos porcentajes distinguen a los conjuntos de CMNI e indican una secuencia de aprovechamiento integral de esta presa.
\end{abstract}

Palabras Clave: Patagonia, Guanaco, Partes Esqueletarias, Marcas de Procesamiento

\section{ABSTRACT}

The objective of this paper is to present the results of the zooarchaeological analysis performed on a series of bone assemblages from cave Milodon North I (CMNI), located in the area of Lake Pueyrredón, Northwest of Santa Cruz, Argentina, that were dated in ca. I,900 years BP.The results point out similarities and differences with other bone sets from other sites in the region with similar chronologies. It is observed, in general, a low diversity of species represented, with an absolute domination of guanaco (Lama guanicoe) remains. The representation of guanaco skeletal parts shows an increased frequency of appendicular elements and a low proportion of ribs, a pattern that does not seem to be totally explained by the action of bone density-dependent processes. The high diversity of processing marks and its high percentages distinguish assemblages of $\mathrm{CMNI}$ and indicate a sequence of comprehensive utilization of this prey.

Key Words: Patagonia, Guanaco, Skeletal Parts, Processing

i CONICET/UBA-Instituto Nacional de Antropología y Pensamiento Latinoamericano. 3 de Febrero 1370, C1426BJN, Ciudad Autónoma de Buenos Aires, Argentina. Correo-e: mariandenigris@yahoo.com.ar

Recibido: 04-12-2012 Revisado: 03-06-2013 Aceptado: 12-12-2013 
Los Conjuntos Óseos de Cueva Milodón Norte I, Lago Pueyrredón (Santa Cruz,Argentina)

\section{INTRODUCCIÓN}

En este trabajo se presentan los resultados de los análisis zooarqueológicos efectuados en las capas 2, 3 y 4 del sitio Cueva Milodón Norte I (CMNI), emplazado a $318 \mathrm{msnm}$ en las cercanías del lago Pueyrredón, noroeste de Santa Cruz, Argentina. Las excavaciones realizadas en este sitio permitieron la identificación de una capa de ceniza volcánica que se corresponde con la erupción del volcán Hudson acaecida hace ca. 6.700 años AP. Se ha registrado la presencia de ocupaciones humanas por encima y por debajo de la tefra, señalando una larga secuencia de ocupación de la cueva, sin embargo, las unidades estratigráficas analizadas en esta ocasión son posteriores a esta erupción, datadas radiocarbónicamente en ca. 1.900 años AP (Capa 4 UGAMS $40191950 \pm 30 ; \delta 13 \mathrm{C}=-22.66$; Aschero et al. 2009). En esta primera etapa fueron estudiados los materiales procedentes de una superficie de $2,25 \mathrm{~m}^{2}$.

Tres aspectos principales son considerados en esta aproximación a los conjuntos de $\mathrm{CMNI}$ : (a) la diversidad taxonómica; (b) la abundancia anatómica de la especie principalmente aprovechada; y finalmente (c) las modificaciones presentes en esa presa.

\section{DIVERSIDAD TAXONÓMICA}

En las tres capas examinadas se observa, en general, una baja diversidad de especies representadas (Tabla I), con un dominio absoluto del guanaco (Lama guanicoe), medido en NISP (Número de Especímenes Identificados por Taxón, Lyman 2008). La segunda categoría más destacada es Artiodactyla. Sin embargo, esta elevada representación se debe a la fragmentación que presentan los especímenes que conlleva a una ausencia de rasgos diagnósticos que hace imposible una adscripción más específica. No obstante, la mayor parte de los especímenes incluidos en este grupo muy probablemente correspondan a guanaco. Las otras especies presentes son aves no determinadas, piche (Zaedyus pichiy) y zorro (Pseudalopex sp.). De éstas solamente el piche registra evidencias de procesamiento humano. Dada esta notable prominencia del guanaco los resultados que se presentan a continuación hacen foco en esta especie.

\begin{tabular}{|l|c|c|c|}
\hline & Capa 2 & Capa 3 & Capa 4 \\
\hline Aves indeterminadas & 5 & 2 & 3 \\
\hline $\begin{array}{l}\text { Piche (Zaedyus } \\
\text { pichiy) }\end{array}$ & 5 & 4 & 8 \\
\hline $\begin{array}{l}\text { Zorro (Pseudalopex } \\
\text { sp) }\end{array}$ & 3 & 1 & 7 \\
\hline Artiodactyla & 146 & 99 & 239 \\
\hline $\begin{array}{l}\text { Guanaco (Lama } \\
\text { guanicoe) }\end{array}$ & 218 & 265 & 257 \\
\hline NISP total & 377 & 371 & 314 \\
\hline
\end{tabular}

Tabla 1: Representación taxonómica (NISP) en Cueva Milodón Norte 1

Table 1: Taxonomic diversity (NISP) in Cueva Milodón Norte 1

\section{ABUNDANCIA ANATÓMICA DE GUANACO}

Para evaluar la abundancia anatómica del guanaco se consideró la relación entre el MNE (Número Mínimo de Elementos, Lyman 2008) observado y el MNE esperado para distintas regiones del esqueleto que fueron consideradas en función de la similitud de recursos que proporcionan (De Nigris 2004). Los datos obtenidos muestran una estrecha semejanza entre los conjuntos de las tres unidades estratigráficas analizadas (Figura I). Todas las regiones anatómicas están presentes indicando el ingreso de esta presa a CMNI de forma completa. Sin embargo, es el esqueleto apendicular el que predomina. El costillar es la región menos representada del segmento axial mientras que el calcáneo y las falanges son las unidades anatómicas menos frecuentes del apendicular. Las extremidades medias -radioulna y tibia-e inferiores -metapodiosson las que predominan en todos los conjuntos. Se evaluó si los patrones observados podrían corresponder a procesos dependientes de la densidad ósea. En ninguno de los casos los resultados de la correlación entre el \%MAU (Lyman 2008) con la densidad ósea (Elkin 1995) superaron los valores medios, por ejemplo se observó un valor de $r_{s}=0,34$ $\mathrm{P}<, 0$ I para la capa 3, aunque en las unidades 2 y 4 $\left(r_{s}=0,44 p>, 05\right.$ y $r_{s}=0,46 p>, 05$ respectivamente) resultaron estadísticamente significativos, indicando que parte de la destrucción podría estar explicada por esta variable. A continuación se consideran los resultados de las modificaciones presentes en los especímenes de guanaco que ayudarán a comprender estos resultados. 


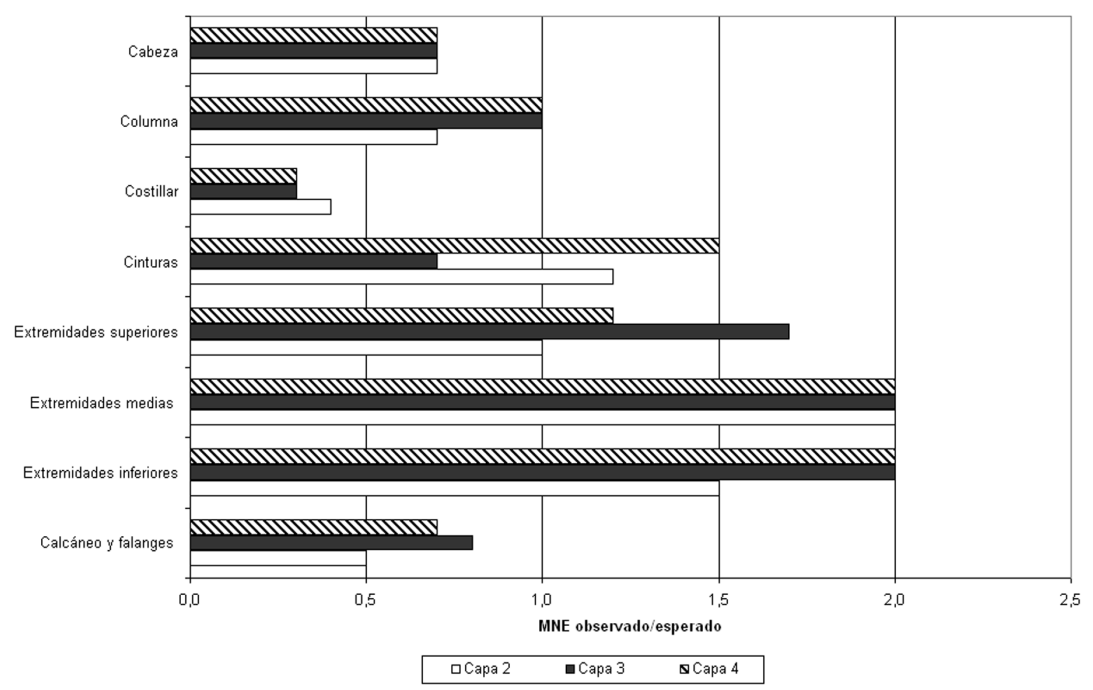

Figura 1: Patrones de representación de unidades anatómicas de guanaco (Lama guanicoe) en Cueva Milodón Norte 1

Figure 1: Anatomical patterns for guanaco (Lama guanicoe) in Cueva Milodón Norte 1

\section{MODIFICACIONES PRESENTES EN GUANACO}

Se evaluaron modificaciones producto de la actividad humana así como la generada por otros agentes y procesos (Tabla 2). Los resultados obtenidos en las tres capas consideradas son nuevamente muy semejantes. Solamente fueron identificadas tres alteraciones de origen no antrópico en los conjuntos estudiados, la meteorización (sensu Behrensmeyer 1978), la acción de roedores y carnívoros (Fisher 1995). La meteorización está presente en todas las unidades estratigráficas analizadas y sus valores oscilan entre un $10 \%$ y un $13 \%$, presentando una distribución de frecuencias bastante semejante entre los distintos estadios. Es posible, entonces, que pequeña parte de la destrucción observada pueda deberse a la acción de este proceso. Las huellas de roedor son predominantes y exhiben porcentajes muy elevados, sin embargo la acción de estos animales no parece haber sido tan destructiva como para contribuir a la fractura ósea. Las alteraciones generadas por los carnívoros son muy escasas y no superan en ninguno de los casos el 3,1\%, siendo observadas, en varios especímenes, en asociación con marcas antrópicas.

\begin{tabular}{|c|c|c|c|}
\hline CMNI & $\begin{array}{c}\text { Capa } 2 \\
(N=2 \mid 0)\end{array}$ & $\begin{array}{c}\text { Capa } 3 \\
(\mathrm{~N}=257)\end{array}$ & $\begin{array}{c}\text { Capa } 4 \\
(\mathrm{~N}=245)\end{array}$ \\
\hline \multicolumn{4}{|l|}{ Modificaciones humanas } \\
\hline Corte & $66(31,4 \%)$ & $78(30,3 \%)$ & $77(31,4 \%)$ \\
\hline Raspado & II (5,3\%) & $10(3,9 \%)$ & $15(6,1 \%)$ \\
\hline Machacado & $4(1,9 \%)$ & $7(2,7 \%)$ & $2(0,8 \%)$ \\
\hline Percusión & $32(15,3 \%)$ & $27(10,5 \%)$ & $26(10,6 \%)$ \\
\hline Marcas de procesamiento (general) & $92(43,8 \%)$ & $106(4 \mid, 2 \%)$ & $104(42,4 \%)$ \\
\hline \multicolumn{4}{|l|}{ Modificaciones no humanas } \\
\hline Roedor & $86(40,9 \%)$ & $112(43,6 \%)$ & $83(33,9 \%)$ \\
\hline Carnívoro & $3(1,4 \%)$ & $8(3,1 \%)$ & $6(2,5 \%)$ \\
\hline Meteorización & $25(11,9 \%)$ & $34(13,2 \%)$ & $26(10,6 \%)$ \\
\hline
\end{tabular}

Tabla 2: Modificaciones óseas en especímenes de guanaco de Cueva Milodón Norte $1^{*}$ Relación entre el NISP total y el NISP con marcas excluyendo los dientes sueltos

Table 2: Bone modification in guanaco specimens of Cueva Milodón Norte $1^{\star}$ Relationship between the total NISP and the NISP with marks excluding loose teeth 
Las marcas de procesamiento y consumo (Fisher 1995), medidas como la relación entre el NISP total y el NISP con marcas, son muy elevadas en todas las capas dominadas por el corte que se ubica alrededor del 30\%, seguidas por la percusión -incluyendo hoyos y estrías- que llega en la unidad I al $15 \%$ y en porcentajes menores por el raspado y el machacado. Estos valores indican que la mayor parte de la destrucción ósea registrada se correspondería con la explotación humana de las carcasas.

\section{DISCUSIÓN}

Los resultados obtenidos señalan una gran similitud entre los tres conjuntos de CMNI estudiados. Se observa en todos los casos al guanaco como el recurso principalmente explotado para el Holoceno tardío lo que indica una gran dependencia en esta presa, aspecto no demasiado llamativo si tenemos en consideración la baja diversidad taxonómica característica de estos ambientes del interior de Patagonia (Redford y Eisenberg 1992).

Asimismo, se observa una representación de las partes esqueletarias de este camélido bastante equilibrada que muestra el ingreso completo de las carcasas al sitio. No obstante, se repite el patrón observado en otros sitios de la estepa patagónica fechados también en el Holoceno tardío, como por ejemplo Cerro de los Indios I en las cercanías del lago Posadas (De Nigris y Catá 2005, Mengoni Goñalons 1999) o Cerro Cuadrado 3 y Cueva de Milodón Norte 2 en el lago Pueyrredón (De Nigris y Tecce 20l3), de una mayor preponderancia de los huesos largos y una baja frecuencia de los costillares (e.g. De Nigris y Mengoni Goñalons 2004). Al respecto es interesante destacar que la escasa proporción de falanges observada en CMNI lo distinguen de las capas más tardías de Cerro de los Indios I o del sitio Cerro Cuadrado 3 (De Nigris y Tecce 2013) y lo acercan a conjuntos con dataciones más tempranas como los de Cerro Casa de Piedra 7 en el Parque Nacional Perito Moreno (De Nigris 2004). En el caso de este último sitio se planteo que la ausencia de estos huesos podría deberse al consumo incidental de estas porciones durante el procesamiento primario de las presas (De Nigris 2004). Es posible entonces que esta situación se repita en los conjuntos de CMNI y que su ausencia no implique necesariamente un falta de aprovechamiento de las fuentes nutritivas -aunque escasas- que proporciona este elemento óseo. Las falanges recuperadas en CMNI, por otra parte, se encuentran en todos los casos fracturadas y con modificaciones producto de la actividad humana indicando que cuando entraban al sitio su médula era consumida.

La elevada diversidad de marcas de procesamiento y sus altos porcentajes caracterizan a los conjuntos de CMNI y se asemejan muchísimo a otros sitios en los que se encuentran representadas todas las etapas del consumo -e.g. Cerro de los Indios I (Mengoni Goñalons 1999) y Cerro Casa de Piedra 7 (De Nigris 2004)- e indican una secuencia de aprovechamiento integral de esta presa acompañada de una destrucción ósea como consecuencia de la explotación de los recursos brindados por el guanaco. La continuación de estos estudios con la incorporación de nuevas variables permitirá continuar caracterizando las ocupaciones más tardías del sitio $\mathrm{CMNI}$.

Agradecimientos: Estas investigaciones fueron financiadas por el proyecto ANPCyT 2488 "Colonización y consolidación en ambientes cordilleranos de Patagonia centro-meridional: área del lago Pueyrredón" dirigido por Carlos Aschero y el UBACyT 20020100200145 "Recursos faunísticos y tecnología lítica en la costa noreste del lago Pueyrredón, Santa Cruz" dirigido por Mariana De Nigris. A María Luz Funes por su invalorable ayuda.

\section{BIBLIOGRAFÍA}

Aschero, C., D. Bozzuto, M. T. Civalero, M. De Nigris, A. Di Vruno, V. Dolce, N. Fernández, L. González y P. Limbrunner. 2009. "El registro arqueológico de la costa noreste del lago Pueyrredón-Cochrane (Santa Cruz, Argentina)". En Arqueología de la Patagonia: una mirada desde el último confin, editado por M. Salemme, F. Santiago, M. Álvarez, E. Piana, M. Vázquez y M. E. Mansur, pp. 919-926. Editorial Utopías, Ushuaia.

Behrensmeyer, A. K. 1978. "Taphonomic and ecologic information from bone weathering”. Paleobiology 4: I50-162.

De Nigris, M. E. 2004. El Consumo en Grupos Cazadores Recolectores. Un Ejemplo Zooarqueológico de Patagonia Meridional. Sociedad Argentina de Antropología, Buenos Aires.

De Nigris, M. E. y M. P. Catá. 2005. "Cambios en los patrones de representación ósea del guanaco en Cerro de los Indios I". Intersecciones en Antropología 6: 109-II 9.

De Nigris, M. E. y G. L. Mengoni Goñalons. 2004. El guanaco como fuente de carne y grasas en Patagonia. En Contra 
viento y marea. Arqueología de Patagonia, editado por M.T. Civalero, P. Fernández y A. G. Guráieb, pp. 469-476. Instituto Nacional de Antropología y Pensamiento Latinoamericano, Buenos Aires.

De Nigris, M. E. y S. Tecce. 2013. "Estudios zooarqueológicos del lago Pueyrredón-Cochrane (Santa Cruz, Argentina)". En Tendencias teórico-metodológicas y casos de estudio en la arqueología de la Patagonia, pp. 335-342, compilado por A. F. Zangrando, R. Barberena, A. Gil, G. Neme, M. Giardina, L. Luna, C. Otaola, S. Paulides, L. Salgán y A.Tivoli. Museo de Historia Natural de San Rafael, San Rafael.

Elkin, D. C. 1995. "Volume density of South american camelid skeletal parts". International Journal of Osteoarchaeology 5:29-37.
Fisher, J.W. 1995. "Bone surface modifications in zooarchaeology". Journal of Archaeological Method and Theory 2(I): 7-67.

Lyman, R. L. 2008. Quantitative Paleozoology. Cambridge University Press, Nueva York.

Mengoni Goñalons, G. L. 1999. Cazadores de guanacos de la estepa patagónica. Sociedad Argentina de Antropología, Buenos Aires.

Redford, K. H. y J. F. Eisenberg. 1992. Mammals of the Neotropics. The Southern Cone.Volume 2: Chile, Argentina, Uruguay, Paraguay. The University of Chicago Press, Chicago. 